\title{
Nonabelian Monopoles and Confinement: New Prospects
}

\author{
Kenichi Konishi*i \\ Univiversity of Pisa, Italy \\ E-mail: konishiedf .unipi.it
}

\begin{abstract}
We discuss quantum mechanical aspects of non abelian monopoles in relation to the phenomena of confinement and dynamical symmetry breaking. In supersymmetric theories there are three types of confining vacua have been found: (i) abelian dual superconductor; (ii) weakly-coupled nonabelian dual superconductor; and (iii) strongly-coupled, nonabelian Argyres-Douglas vacua. The last class of systems occur very generally in supersymmetric theories; they are characterized by strongly-coupled nonabelian monopoles and dyons: confinement and dynamical symmetry breaking are caused by the condensation of monopole composites, rather than by condensation of single weakly-coupled monopoles. There are strong constraints on which kind of monopoles can appear as the infrared degrees of freedom, related to the proper realization of the global symmetry of the theory. Drawing analogies to some of the phenomena found here, we make a speculation on the ground state of the standard QCD.
\end{abstract}

International Europhysics Conference on High Energy Physics

July 21st - 27th 2005

Lisboa, Portugal

\footnotetext{
* Speaker.

${ }^{\dagger}$ Based on the work in collaboration with G. Marmorini and N. Yokoi [1].
} 


\section{Introduction}

Our understanding of the quantum behavior of nonabelian monopoles in $4 \mathrm{D}$ gauge theories has greatly improved over the last few years. By exploiting the knowledge of the exact solutions in theories with $\mathscr{N}=1$ or $\mathscr{N}=2$ supersymmetry we know for instance that there are systems in which the 't Hooft-Mandelstam scenario of confinement (dual Meissner effect) is indeed realized dynamically. However, in all systems of this type confinement is accompanied by dynamical abelianization, a feature which is not shared by quantum chromodynamics (QCD).

Actually, a more general class of supersymmetric theories with massless flavors exhibit a rather different picture of confinement and dynamical symmetry breaking. First of all, confinement is typically described as a dual Meissner effect, of a nonabelian kind, where both condensing monopoles and confining strings carry unbroken nonabelian fluxes. The properties of these nonabelian superconductors are being intensely investigated.

Of particular interest is the class of vacua in which confinement and dynamical symmetry breaking are caused by strongly interacting monopoles and dyons. The theory is close to an infrared fixed point - a nontrivial superconformal theory. Although these systems defy the traditional effective-Lagrangian approach, it is still possible to get a fairly precise picture of what is happening [1], by fully mobilizing our general knowledge such as the Nambu-Goldstone theorem, SeibergWitten curves, instanton-induced effective action, holomorphic properties due to supersymmetry, vacuum counting, decoupling theorem, exact anomalous and non-anomalous Ward-Takahashi identities, universality of conformal theories, Seiberg's duality, some new results on $\mathscr{N}=1$ susy gauge theories, and so on.

\section{Argyres-Douglas vacua}

An interesting class of superconformal theories which arise as infrared fixed point was discovered by Argyres and Douglas, and by others. The first example studied is a pure $\mathscr{N}=2$ supersymmetric $S U$ (3) Yang-Mills theory: the theory flows in the infrared into an effective $U(1) \times U(1)$ theory, where one of the $U(1)$ factor is a strongly-coupled nontrivial fixed point theory. Interest in this kind of systems (which we shall call Argyres-Douglas vacua below) arises from the fact that, in contrast to the cases discussed by Seiberg and others, the nature of the low-energy degrees of freedom is somewhat better understood and, in particular, because they are known to include a mutually nonlocal set of dyon fields. In the specific case of the interacting $U(1)$ SCFT of Argyres and Douglas, the IR "matter" degrees of freedom are a magnetic monopole, a dyon and an electron. The beta function cancel among the contributions from these relatively nonlocal fields.

\section{3. $S U(N)$ theories}

In the $\mathscr{N}=2 S U(N) \mathrm{SQCD}$, the degrees of freedom in UV are (in $\mathscr{N}=1$ formalism) the gauge multiplet $W=\left(A_{\mu}, \lambda\right)$, the adjoint chiral multiplet $\Phi=(\phi, \psi)$, and the quark multiplets $Q_{i}=$ $\left(q, \psi_{Q}\right)_{i}$ and $\tilde{Q}_{i}=\left(\tilde{q}, \tilde{\psi}_{Q}\right)_{i}$, in the fundamental (antifundamental) representation of the gauge group $\left(i=1,2, \ldots, N_{f}\right)$. We start with small quark masses $m_{i}$ and we break the $\mathscr{N}=2$ supersymmetry to 
$\mathscr{N}=1$ by a small superpotential (mass term) for the adjoint chiral multiplet,

$$
\left.\mu \operatorname{tr} \Phi^{2}\right|_{\mathrm{F}}=\mu \psi \psi+\ldots
$$

The simplest nonabelian Argyres-Douglas vacuum appears in the $S U(3)$ gauge theory with $N_{f}=4$ flavors. The low-energy effective gauge group is $S U(2) \times U(1)$. If the masses $m$ are large compared to the dynamical scale of the theory $\Lambda$, the theory is basically the local $S U(2)$ theory with $N_{f}=4$ flavors, which as is well known, is conformally invariant, with $\beta=0$. As $m \rightarrow 0$, however, the flow of the theory into the infrared fixed point occurs in a nontrivial way. The low-energy physics of $\mathscr{N}=1$ vacua are encoded in the structure of the singularities of the Seiberg-Witten curve, which in this case reads,

$$
y^{2}=\prod_{a=1}^{3}\left(x-\phi_{a}\right)^{2}-\prod_{i=1}^{4}\left(x+m_{i}\right) \equiv\left(x^{3}-U x-V\right)^{2}-4 \Lambda^{2} \prod_{i=1}^{4}\left(x+m_{i}\right),
$$

where $U=\frac{1}{2}\left\langle\operatorname{tr} \Phi^{2}\right\rangle$ and $V=\frac{1}{3}\left\langle\operatorname{tr} \Phi^{3}\right\rangle$ parametrize inequivalent vacua. A careful study shows that the low-energy degrees of freedom are found to carry the magnetic and electric $U(1) \times U(1)$ charges, shown in Table 1, with the first $U(1)$ factor (magnetic or electric) referring to the subgroup of the $S U(2)$. The system having $\mathscr{N}=2$ supersymmetry, there are also particles $\tilde{M}^{\alpha}, \tilde{D}^{\alpha}, \tilde{E}^{\alpha}$, with conjugate gauge quantum numbers. Having this knowledge, and knowing how the global symmetry

\begin{tabular}{|l|l|}
\hline Particles & $\left(g_{1}, g_{2} ; q_{1}, q_{2}\right)$ \\
\hline$M_{1}, M_{2}$ & $( \pm 1,1 ; 0,0)^{4}$ \\
$D_{1}, D_{2}$ & $( \pm 2,-2 ; \pm 1,0)$ \\
$E_{1}, E_{2}$ & $(0,2 ; \pm 1,0)$ \\
\hline
\end{tabular}

Table 1: The charges of the massless doublets. $g_{i}\left(q_{i}\right)$ is the magnetic (electric) charge with respect to the $i$-th $U(1)$ factor.

is broken at large $\mu$, we conclude that the baryonlike condensates

$$
\left\langle\varepsilon_{\alpha \beta} M_{i}^{\alpha} M_{j}^{\beta}\right\rangle \neq 0, \quad\left\langle\varepsilon^{\alpha \beta} \tilde{M}_{\alpha}^{i} \tilde{M}_{\beta}^{j}\right\rangle \neq 0,
$$

form, leading to confinement and symmetry breaking.

In more general $r=\frac{N_{f}}{2}$ vacua of $S U(N)$ theory, one expects again that nonabelian monopoles $M_{i}^{\alpha}$ in the fundamental representation of $S U\left(\frac{N_{f}}{2}\right)$ gauge group and in the fundamental representation of the global $S U\left(N_{f}\right)$ group, will be responsible for confinement/dynamical symmetry breaking. From the known symmetry breaking pattern

$$
S U\left(N_{f}\right) \times U(1) \rightarrow U\left(\frac{N_{f}}{2}\right) \times U\left(\frac{N_{f}}{2}\right),
$$

we conclude that the baryonlike condensate

$$
\left\langle\varepsilon_{\alpha_{1} \alpha_{2} \ldots \alpha_{N_{f} / 2}} M_{i_{1}}^{\alpha_{1}} M_{i_{2}}^{\alpha_{2}} \ldots M_{i_{N_{f} / 2}}^{\alpha_{N_{f} / 2}}\right\rangle \neq 0
$$

forms in this vacuum. 


\section{4. $U S p(2 N)$ theories}

Another simple but very instructive example of nonabelian Argyres-Douglas vacua occurs in $U S p(4)$ theory with $N_{f}=4$ flavors. A characteristic feature of this model, which makes it really interesting, as compared to the $S U(N)$ theories discussed above, is the fact that it possesses a nontrivial chiral symmetry, $G_{F}=S O(8)$. This system has been carefully studied, by following the method used for the previous case, by Auzzi and Grena. Their result is summarized in the following table. They find that, as compared to the $S U$ (3) SCFT considered above, there is one extra doublet in this system $\left(C_{1}, C_{2}\right.$ in Table. 2). Given the charges of the massless particles and

\begin{tabular}{|l|l|}
\hline Particles & Charge \\
\hline$M_{1}, M_{2}$ & $( \pm 1,1,0,0)^{4}$ \\
$D_{1}, D_{2}$ & $( \pm 2,-2, \pm 1,0)$ \\
$E_{1}, E_{2}$ & $(0,2, \pm 1,0)$ \\
$C_{1}, C_{2}$ & $( \pm 2,0, \pm 1,0)$ \\
\hline
\end{tabular}

Table 2: The charges of the massless doublets in one of the SCFT vacua.

given the symmetry breaking pattern, $S O(8) \rightarrow U(4)$, (known from the analysis at large $\mu$, we are forced to conclude that the monopole pair $M, \tilde{M}$ condenses as

$$
\left\langle M_{a}^{i} \tilde{M}_{j}^{a}\right\rangle=v \delta_{j}^{i} \neq 0, \quad a, b=1,2 \quad \text { and } \quad i, j=1, \ldots, 4 .
$$

All of the confining vacua of $U S p(2 N)$ theories with $m_{i} \rightarrow 0, \mu \neq 0$, with $N_{f} \neq 0$ flavors, are perturbation of nontrivial superconformal vacua, as in the $U S p(4)$ example discussed above. We expect that mesonlike condensates of strongly interacting nonabelian monopoles form in such systems, causing confinement and at the same time dynamical symmetry breaking.

\section{Conclusion}

To conclude, quantum behavior of nonabelian monopoles turn out to be quite weird, subtle, and yet consistent. There are in general strong constraints arising from the requirement, that the symmetries of the underlying theory is appropriately realized in low energies, on which types of monopoles can appear as infrared degrees of freedom. GNOW monopoles do not always survive quantum effects. Appearance of the dual group crucially depends on the massless flavors.

Nonabelian Argyres-Douglas type vacua appear to be the best candidate for QCD. By relying heavily on analogy with the cases found in supersymmetric theories, we might speculate on the vacua on QCD characterized by the facts: (i) the theory is close to SCFT (relatively nonlocal dyons); (ii) confinement caused by strongly-coupled (nonabelian) monopoles; (iii) chiral symmetry breaking induced by the same composites monopole condensate.

\section{References}

[1] G. Marmorini, K. Konishi and N. Yokoi, "Nonabelian confinement near nontrivial conformal vacua", [arXiv: hep-th/0511121], and references therein. 